\title{
The study of temperature dependence of photoresponse in superlattice infrared photodetectors
}

\author{
J. H. Lu, Y. C. Wang, C. L. Wang, and C. H. Kuana) \\ Department of Electrical Engineering, National Taiwan University, Taipei, Taiwan 10617, \\ Republic of China and Graduate Institute of Electronics Engineering, National Taiwan University, Taipei, \\ Taiwan 10617, Republic of China \\ C. W. Yang and S. L. Tu \\ Opto Tech Corporation, Hsinchu, Taiwan 310, Republic of China \\ J. Y. Feng and T. S. Lay \\ Institute of Electro-Optical Engineering, National Sun Yat-Sen University, Kaohsiung, Taiwan 804, \\ Republic of China
}

(Received 1 March 2007; accepted 5 August 2007; published online 2 October 2007)

\begin{abstract}
Temperature dependence of the photoresponse in two superlattice infrared photodetectors with different single barriers is studied. It is found that the short-wavelength response decreases with temperature, but the long-wavelength one increases. The crossing point of those two response ranges is independent of temperature. The movement of its associated wavelength under different biases is investigated and analyzed. The relevant physical mechanisms are found and integrated into a simple model to explain the experimental results. Based on the model, another sample with a superlattice integrated with multiple quantum wells is designed to demonstrate whether its temperature dependence of the photoresponse is consistent with our understanding. The sample indeed shows a broadband response which enhances with temperature in all wavelengths. (C) 2007 American Institute of Physics. [DOI: 10.1063/1.2785013]
\end{abstract}

\section{INTRODUCTION}

The focal plane array (FPA) imaging systems based on quantum well infrared photodetectors (QWIPs) have shown the potential use for military, medical, and civil applications. ${ }^{1-4}$ However, the main drawback of the QWIPs under high temperature $(T)$ operation is the large dark current. Although many theoretical and experimental studies on the $T$ effect on QWIP performances have been carried out, ${ }^{5-13}$ the similar research in another promising detector named as superlattice infrared photodetectors (SLIPs) is deficient. In this paper, we study the $T$ dependence of the photoresponse in SLIPs and design a structure to have better photoresponse enhancement when temperature rises.

The structure of an ordinary SLIP is a superlattice (SL) integrated with a single barrier. The functions of the single barrier are not only to reduce the dark current but also to act as a bias-tuned energy filter. ${ }^{14,15}$ The electron transport through the barrier is dependent on two factors: the barrier thickness and the externally applied bias. The thinner barrier allows electrons to cross with a minimized chance for the scattering which may increase with the applied bias. ${ }^{15}$ Therefore, electron transport in a thin-barrier sample under low bias operation is likely to be ballistic, and the associated photocurrent loss caused by the scattering is expected to be reduced even under high- $T(80 \mathrm{~K})$ operation.

For the SL itself, the electron distribution at each state in the first miniband $\left(M_{1}\right)$ is decided by Fermi level $\left(E_{F}\right)$ and $T$ simultaneously. Meanwhile, the $E_{F}$ position can be con-

\footnotetext{
${ }^{a)}$ Tel.: $\quad 886-2-3366-3569 . \quad$ FAX: $886-2-2368-1679 . \quad$ Electronic mail: kuan@cc.ee.ntu.edu.tw
}

trolled by the doping density in the wells of the SL. Hence the SLIPs with different doping densities will have different photoresponse spectra because the photocurrent contribution from each state depends on the $E_{F}$ position.

From above descriptions, it is concluded that the factors such as the doping density, the single-barrier's thickness, and the externally applied bias are possible to affect the photoresponse of a SLIP at different $T$. In this paper, we will present the effects of the doping density and the barrier thickness associated with $T$ in Figs. 2 and 3 and the influence of applied bias in Figs. 4 and 5. By integrating these results, we try to propose a simple model to explain the $T$ dependence of photoresponse in SLIPs. Based on the model, a detector is designed to demonstrate whether the $T$ dependence of photoresponse is consistent with our expectations. The related experimental result is shown in Fig. 6.

\section{SAMPLE STRUCTURE}

Two samples, A and B, with the same 15-period SL structure but different single-barrier thicknesses as shown in Fig. 1 are investigated at first. The single barriers of samples $\mathrm{A}$ and $\mathrm{B}$ are 300 and $50 \mathrm{~nm} \mathrm{Al}_{0.25} \mathrm{Ga}_{0.75} \mathrm{As}$, respectively. Each period of the SL consists of $6.5 \mathrm{~nm} \mathrm{GaAs}\left(N_{d}=5\right.$ $\times 10^{17} \mathrm{~cm}^{-3} \mathrm{Si}$ ) and $3.5 \mathrm{~nm} \mathrm{Al} \mathrm{Al}_{0.32} \mathrm{Ga}_{0.68} \mathrm{As}$ (undoped). The above mentioned structures are sandwiched between two GaAs contact layers. The absorption wavelength of the SL is $6-10 \mu \mathrm{m}$. It is also indicated in Fig. 1 that because of the selection rule and momentum conservation, the electrons at $E_{1, n}$, i.e., the $n$th state in $M_{1}$, can only transit to $E_{2,16-n}$, i.e., the $(16-n)$ th state in $M_{2}$ where $n=1-15$ for a 15 -period SL and $n=1$ means the lowest state. 


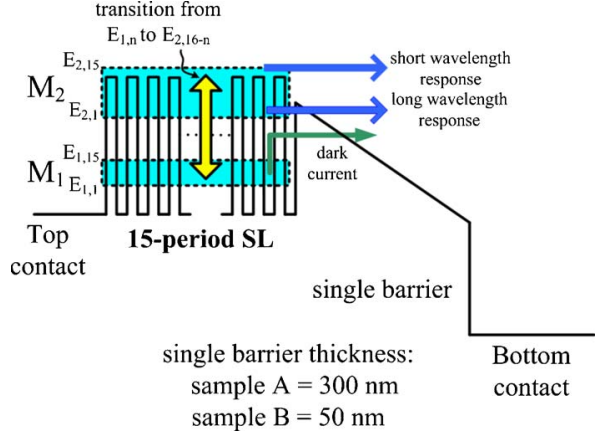

FIG. 1. (Color online) Schematic band diagrams of samples A and B.

\section{RESULTS AND DISCUSSION}

Figure 2 shows the photoresponse spectra of sample A under $0.5 \mathrm{~V}$ at different $T$ and the inset shows the enlarged ones at wavelength of 7-8 $\mu \mathrm{m}$. It is observed that the longwavelength response enhances with $T$, while the shortwavelength one decreases. Similar results are observed in sample B. The above observations are attributed to the change of the electron distribution in $M_{1}$ when $T$ rises. According to our doping density in the SL, the $E_{F}$ position is $55 \mathrm{meV}$ above the GaAs conduction band and lies approximately in the middle of $M_{1}$. The electron distribution at $E_{1, n}$ at $T$ is given by the Fermi-Dirac (FD) distribution function,

$$
f_{T}\left(E_{1, n}\right)=\frac{1}{1+\exp \left(\frac{E_{1, n}-E_{F}}{k T}\right)} .
$$

It indicates that the electron population at the states higher (lower) than $E_{F}$ increases (decreases) with $T$. And this leads to the enhancement (reduction) of the long (short) wavelength response as $T$ rises.

It is also noted in Fig. 2 that those response spectra at different $T$ happen to cross at the same point. In order to further clarify the $T$ dependence of photoresponse, we subtract the photoresponse at $20 \mathrm{~K}$ from that at other higher temperatures to obtain the responsivity difference between these two temperatures. Meanwhile, the crossing point is also shifted to zero. In the rest of this paper, we will observe the variation of the responsivity difference with $T$ or other parameters to understand the $T$ dependence of the photoresponse in our detectors.

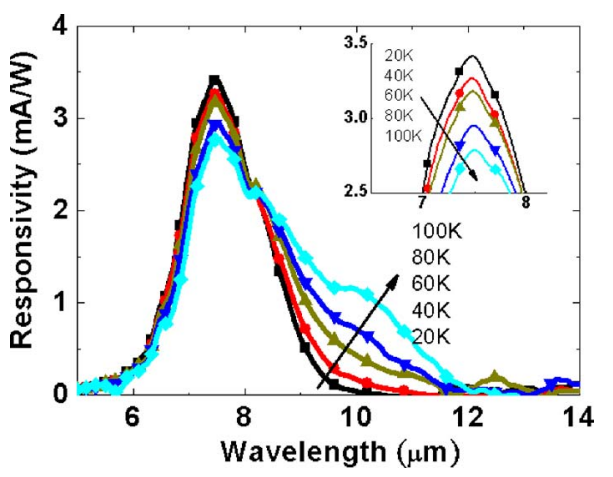

FIG. 2. (Color online) Photoresponse of sample A at different $T$ under $0.5 \mathrm{~V}$.

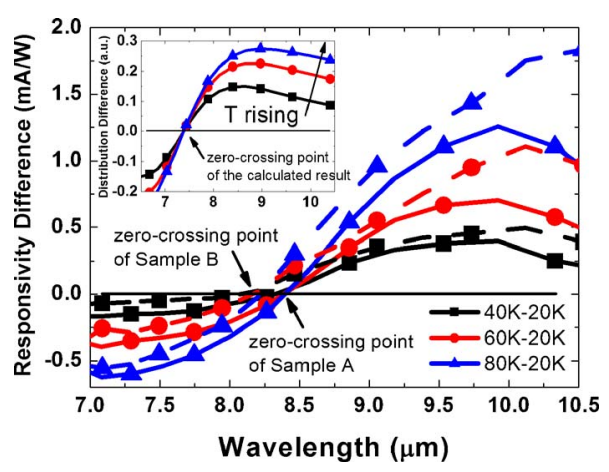

FIG. 3. (Color online) Responsivity difference between $20 \mathrm{~K}$ and other temperatures of samples A (solid lines) and B (dashed lines) under the same electric field. The inset shows the calculated FD distribution difference between $20 \mathrm{~K}$ and other temperatures by Eq. (1).

The responsivity differences versus wavelength between $20 \mathrm{~K}$ and other temperatures for samples A and B are shown with solid and dashed lines in Fig. 3, respectively. It is noted that the two parts, no matter positive or negative one, both become more apparent as $T$ rises. In Fig. 3, the applied biases on samples A and B are 0.6 and $0.1 \mathrm{~V}$, respectively. Because the SL is a low-resistance structure, it is expected that no bias voltage drops across the SL, and hence the barriers of these two samples are under the same electric field. With Eq. (1), the calculated FD distribution differences between $20 \mathrm{~K}$ and the other temperatures are also sketched in the inset of Fig. 3. In the inset, $f_{T}\left(E_{1, n}\right)$ in Eq. (1) has been transformed into $f_{T}\left(\lambda_{n}\right)$, where $\lambda_{n}$ is the transition wavelength from $E_{1, n}$ to $E_{2,16-n}$, as indicated in Fig. 1 and is calculated with $\lambda_{n}=1.24 /\left(E_{2,16-n}-E_{1, n}\right)$. The calculated curves show a consistent trend with the experimental results. However, the positions of the zero-crossing points are different. The wavelength of the calculated result is shorter than those of both samples. This discrepancy will be discussed in Figs. 4 and 5 later.

From Fig. 3, we can also observe the influence of the barrier thickness. Sample B has the magnitude of the positive (negative) part to be larger (smaller) than that of sample A. It is attributed to the smaller scattering loss in the thinner single barrier of sample B. The electron in sample B is more likely to transport through the single barrier than sample A. More detailed analysis will be given in our model.

In this section, the effect of the externally applied bias is presented. The responsivity differences between 80 and $20 \mathrm{~K}$ of samples A and B under different applied biases are shown in Figs. 4(a) and 5(a), respectively. As the bias increases, the zero-crossing points shift to the longer wavelength, and the magnitudes of the positive and negative parts of the responsivity difference are both raised. The applied bias is found to affect the zero-crossing position and may be the primary cause of the discrepancy mentioned in Fig. 3. In order to understand the movement of the zero-crossing point and the magnitudes of both parts versus the applied bias, other possible physical mechanisms including the FD distribution used in Fig. 3 are going to be found and integrated into a simple model in the following.

As shown in Fig. 1, the photoelectrons are excited from $E_{1, n}$ to $E_{2,16-n}$ and travel in $M_{2}$. It is noted that they should 


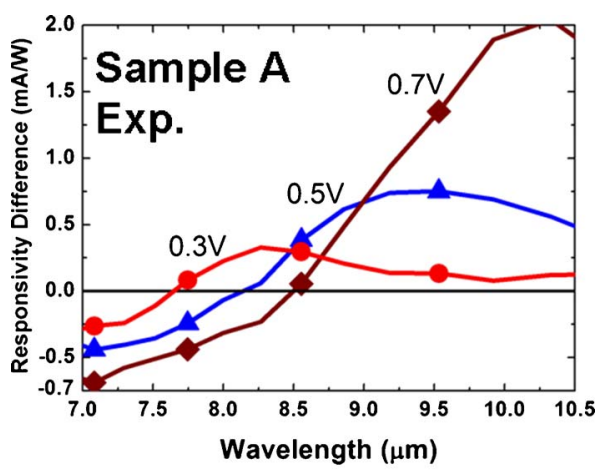

(a)

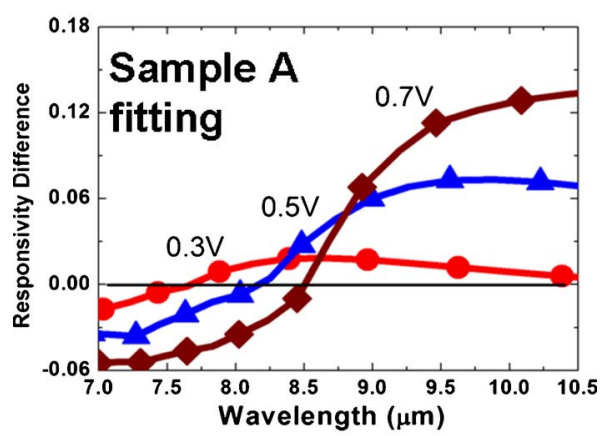

(b)

FIG. 4. (Color online) (a) Responsivity difference between 20 and $80 \mathrm{~K}$ at different biases of sample A. (b) Simulation result of sample A calculated by Eq. (2).

suffer the intraminiband relaxation (not shown in the figure) to cause the redistribution before they arrive at the interface between the SL and the single barrier. It is expected that almost all photoelectrons attempt to accumulate at the lower states in $M_{2}$. We assume that for photoelectrons excited to $E_{2,16-n}$, then after redistribution, their population at $E_{2,15-n}$ is $\gamma$ times more than that at $E_{2,16-n}$, the population at $E_{2,14-n}$ is also $\gamma$ times more than that at $E_{2,15-n}$, and so on. As a result, the distribution at each state in $M_{2}$ is like a geometric series. This assumption is tried to simulate the redistribution and may not be in complete agreement with the detailed population. In addition, $\gamma$ is also assumed to be $T$ independent since the dominant mechanism to cause the intraminiband relaxation is the carrier-carrier scattering.

Also shown in Fig. 1, the energy height of the single barrier is designed to be higher than $E_{2,1}$. After the redistribution, the photoelectrons at the lower states of $M_{2}$ than the barrier height have to tunnel through the interface between the SL and single barrier with the assistance of the electric field. Those at the states higher than the barrier height just pass over the interface with the tunneling probability of 1 . Hence a tunneling probability depending on the energy state of the photoelectrons will be included to describe their transport through the interface between the SL and single barrier.

Besides, the scattering loss of the photoelectron population during their transport through the single barrier is also considered in our model, in particular, to understand the shift of zero-crossing points with the applied bias. The scattering leads to a decrement of the photoelectron population, and we assume that it increases with the applied electric field ${ }^{16}$ and

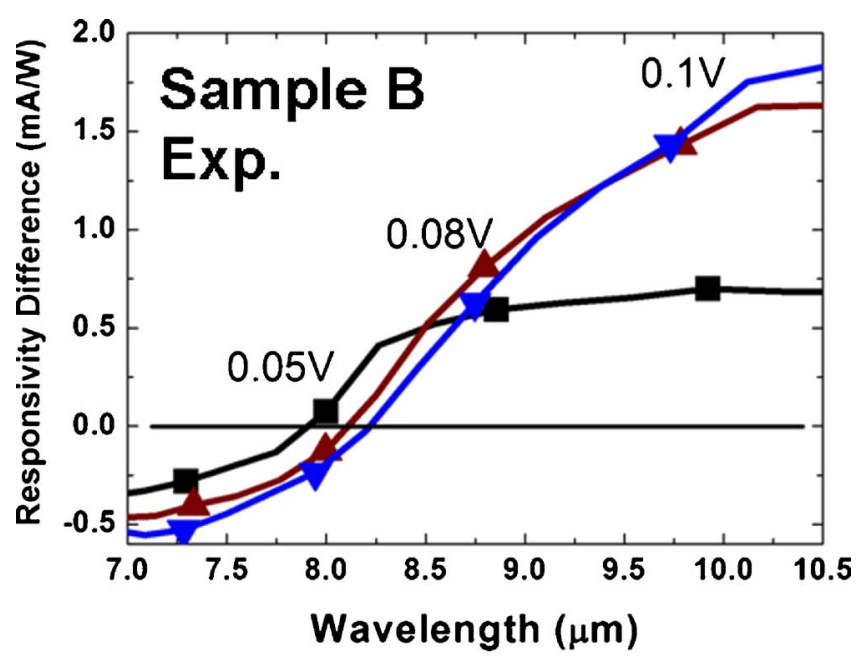

(a)

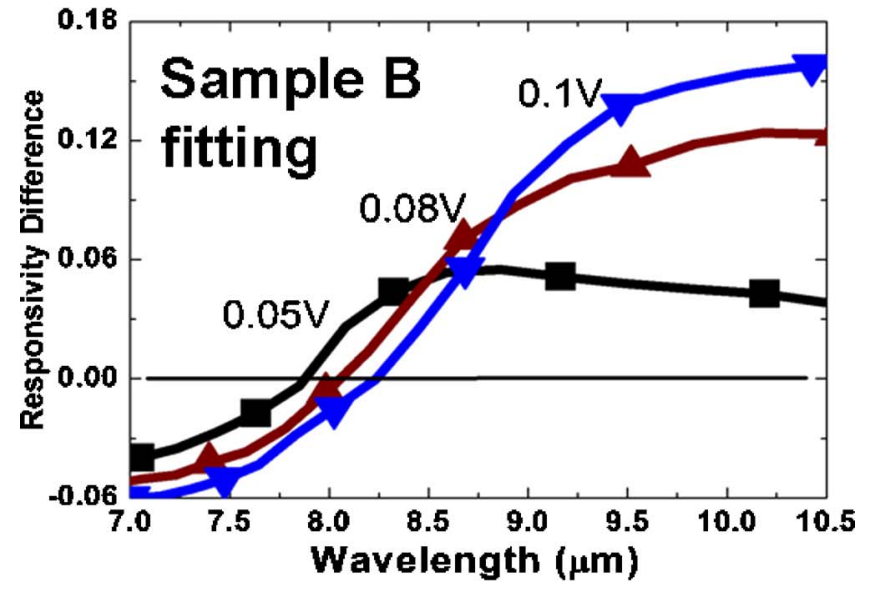

(b)

FIG. 5. (Color online) (a) Responsivity difference between 20 and $80 \mathrm{~K}$ at different biases of sample B. (b) Simulation result of sample B calculated by Eq. (2).

the temperature since the dominant mechanisms are the intervalley scattering and the phonon scattering which are related to the bias and temperature, respectively.

All above mechanisms can be integrated into one equation,

$$
\begin{aligned}
D\left(\lambda_{n}, F\right)= & {\left[f_{80 \mathrm{~K}}\left(\lambda_{n}\right)-f_{20 \mathrm{~K}}\left(\lambda_{n}\right)\right] \sum_{i=1}^{16-n} } \\
& \times\left[\left(\frac{\gamma^{j-1}}{1+\gamma+\gamma^{2}+\cdots+\gamma^{15-n}}\right) T\left(E_{2,17-n-i}, F\right)\right] \\
& -(\alpha F+\beta),
\end{aligned}
$$

where $D\left(\lambda_{n} F\right)$ represents the population difference at wavelength $\lambda_{n}$ under electric field $F$ and $f_{80 \mathrm{~K}}\left(\lambda_{n}\right)$ and $f_{20 \mathrm{~K}}\left(\lambda_{n}\right)$ are the FD distribution functions at 80 and $20 \mathrm{~K}$, respectively. The population difference will be used to simulate the responsivity difference shown in Figs. 4 and 5 with the velocity effect neglected for simplicity. In the right-hand side of Eq. (2), the first term is the FD distribution difference used in Fig. 3. The second term is the contribution of each state in $M_{2}$, accounts for the intraminiband relaxation, and 


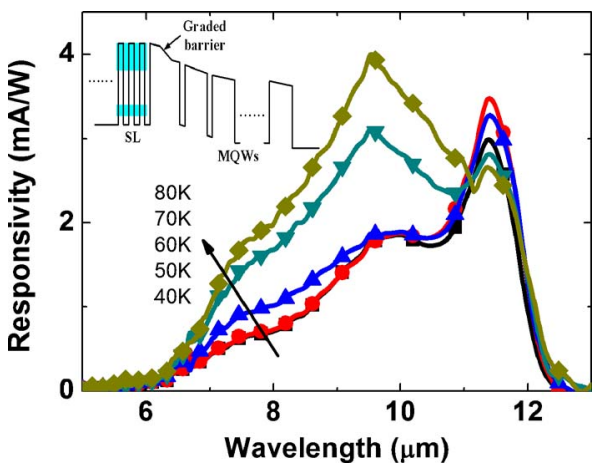

FIG. 6. (Color online) Photoresponse of sample $\mathrm{C}$ at different $T$ under $-0.5 \mathrm{~V}$. The inset shows its band diagram.

has the normalization factor $\left(1+\gamma+\ldots+\gamma^{15-n}\right)$ to keep the total population a constant. The relaxation is assumed to be $T$ independent since the dominant mechanism is the carriercarrier scattering. $T\left(E_{2,17-n-i}, F\right)$ is the Wentzel-KramersBrillouin (WKB) approximation for the tunneling probability $^{15}$ of photoelectrons at $E_{2,17-n-i}$ under $F$. For the scattering loss in the single barrier, we assume that it is a first-order approximation in the form of $\alpha F+\beta$, where $\alpha$ and $\beta$ are constants and unrelated to the electric field. Actually, the relaxation loss between the minibands of SL is also contained in the $\beta$ parameter. The negative sign before $\alpha F+\beta$ stands for a larger loss at $80 \mathrm{~K}$ than $20 \mathrm{~K}$, which is attributed to the phonon scattering. In addition, the scattering loss is also assumed to be independent of the electron injection energy in $M_{2}$ because of the small energy range of $M_{2}$. All the assumptions are utilized to simplify the calculation in order to understand the relations between the mentioned mechanisms and the photoresponse.

The best fitting results between Eq. (2) and the results of sample A shown in Fig. 4(a) or those of sample B shown in Fig. 5(a) all give $\gamma=1.3$. The values of $\alpha$ and $\beta$ for sample A are 0.035 and 0.005 , while those for sample B are 0.01 and 0.002 . The simulation results calculated by Eq. (2) for samples A and B are plotted in Figs. 4(b) and 5(b), respectively. These curves show the agreement with the experimental results. It is also noted that the values of $\alpha$ and $\beta$ can fit all the curves and are independent of $T$. The $\alpha$ factor indicates that the scattering loss may also be attributed to the intervalley scattering. ${ }^{17,18}$ The smaller fitting parameters in sample B may mean a smaller loss because of the thinner barrier.

From the fitting curves with Eq. (2), we can further understand the detailed physical mechanisms. The doping density in the SL wells decides the Fermi level position, the FD distribution, and the initial zero-crossing position. The electron redistribution and barrier height affect the magnitude of positive and negative parts of the responsivity difference but are independent of the shift of the zero-crossing position. The influences of the barrier thickness and applied bias are the scattering loss and also the shift of zero-crossing points.

With the above understanding, we designed sample $\mathrm{C}$ whose structure is shown in the inset of Fig. 6. It contains a 15-period SL, a $60 \mathrm{~nm} \mathrm{Al}_{x} \mathrm{Ga}_{1-x}$ As graded barrier $(x=0.29$ for $20 \mathrm{~nm}, x=0.29$ to $x=0.21$ for $20 \mathrm{~nm}$, and $x=0.21$ for
$20 \mathrm{~nm}$ ), and a 50-period multiple quantum wells (MQWs). The MQWs and the SL structures are, respectively, $6 \mathrm{~nm}$ GaAs $\left(N_{d}=4 \times 10^{17} \mathrm{~cm}^{-3} \mathrm{Si}\right) / 50 \mathrm{~nm} \quad \mathrm{Al}_{0.21} \mathrm{Ga}_{0.79} \mathrm{As}$ (undoped $)$ and $6 \mathrm{~nm}$ GaAs $\left(N_{d}=4 \times 10^{17} \mathrm{~cm}^{-3} \mathrm{Si}\right) / 4 \mathrm{~nm}$ $\mathrm{Al}_{0.29} \mathrm{Ga}_{0.71} \mathrm{As}$ (undoped). The absorption wavelength of the SL is $6-10 \mu \mathrm{m}$. The transition type of the MQWs is bound to bound, and its longest absorption wavelength is about $11.7 \mu \mathrm{m}$. The different absorption ranges of the SL and the MQWs are designed for the broadband response. The MQW is also to act as a noise filter, and the associated mechanism and detailed performance of sample $\mathrm{C}$ are presented in our paper. $^{19}$

The design principles of sample $\mathrm{C}$ are as follows. Firstly, we lower the doping density in sample $\mathrm{C}$ to move the initial zero-crossing point toward the short wavelength. Meanwhile, the detector is also designed to operate at a low bias with the zero-crossing point, not shifting much to the long wavelength. As a result, it is expected that almost whole response spectrum at a low bias can enhance with $T$ to achieve the broadband detection at high temperature. Secondly, we raise the graded barrier height to reduce the dark current at high $T$ and keep its width as $60 \mathrm{~nm}$ to have a high responsivity. Because the photoelectrons have higher energy than the dark electrons, the decrement of the photocurrent due to the raised barrier should be less sensitive. Besides, the barrier thickness of MQWs is $10 \mathrm{~nm}$ thinner than that of the graded barrier. Although the total thickness of the MQWs is much thicker, the electrons can traverse only one barrier each time under the low bias because of the high capture probability ${ }^{18}$ of the wells. The MQWs therefore can be regarded as 50 identical single barriers in series. Except the graded barrier, the equivalent single barrier width is $50 \mathrm{~nm}$, which is thin enough to keep the scattering loss to be small.

Figure 6 shows the photoresponse of sample $\mathrm{C}$ under $-0.5 \mathrm{~V}$, where the $6-10 \mu \mathrm{m}$ response and $11.4 \mu \mathrm{m}$ peak are attributed, respectively, to the SL and the MQWs. It is observed that the SL responses are almost unchanged at low $T$ $(\leqslant 50 \mathrm{~K})$, but increase dramatically once $T$ is higher than $60 \mathrm{~K}$. In comparison with the single-barrier samples, sample $\mathrm{C}$ has better enhancement because the whole spectrum increases with $T$. At the same time, sample $\mathrm{C}$ also demonstrates the broadband detection at $T$ higher than $70 \mathrm{~K}$. It is noted that the MQW response decreases slightly when SL response begins to increase at high $T$. This is because the photoelectrons injected from the SL are dominant in the total photocurrent at high $T$. Under a low bias, the photoelectrons injected from the SL are captured and reemitted consecutively by each well in the MQWs. As $T$ rises, their number increases and the resulted bias on the graded barrier must be increased to push more photoelectrons to pass the MQWs. Because the total bias is fixed, the bias on the other barriers and the response of the MQWs are both hence decreased. This is also the same reason why the MQWs can be considered as 50 identical single barriers in series.

\section{CONCLUSION}

In conclusion, it is found that the $T$ dependence of the photoresponse in SLIPs is affected by four factors including 
the doping density in SL, the externally applied bias, the single-barrier height, and thickness. The doping density decides the Fermi level position in SL and the initial zerocrossing position of the responsivity difference. The applied bias determines the tunneling probability and the scattering loss during the electron transport through the single barrier. The single-barrier height affects the tunneling mechanism as well and the magnitude of positive and negative parts of the responsivity difference. The single-barrier thickness also concerns the scattering loss. Based on the mechanisms affected by those factors, a simple model is established and the resulted simulation shows the consistency with the experimental results. We also design a sample according to the understood mechanisms, and the experimental results show agreement with our design principles.

\section{ACKNOWLEDGMENT}

This work was supported by National Science Council of the Republic of China under Contract No. NSC 94-2215-E002-017.

${ }^{1}$ S. D. Gunapala et al., Semicond. Sci. Technol. 20, 473 (2005).

${ }^{2}$ S. D. Gunapala, S. V. Bandara, J. K. Liu, C. J. Hill, S. B. Rafol, and J. M. Mumolo, IEEE Trans. Electron Devices 50, 2353 (2003).

${ }^{3}$ H. Schneider et al., Infrared Phys. Technol. 47, 53 (2005).
${ }^{4}$ J. Jiang, S. Tsao, K. Mi, M. Razeghi, G. J. Brown, C. Jelen, and M. Z. Tidrow, Infrared Phys. Technol. 46, 199 (2005).

${ }^{5}$ G. Gumbs, D. Huang, and V. Fessatidis, J. Appl. Phys. 75, 7942 (1994).

${ }^{6}$ A. Majumdar, K. K. Choi, L. P. Rokhinson, and D. C. Tsui, Appl. Phys. Lett. 82, 686 (2003).

${ }^{7}$ X. D. Jiang, S. S. Li, and M. Z. Tidrow, IEEE J. Quantum Electron. 35, 1685 (1999)

${ }^{8}$ C. H. Chu, C. I. Hung, Y. H. Wang, and M. P. Houng, IEEE Photonics Technol. Lett. 9, 1262 (1997).

${ }^{9}$ H. Schneider, C. Schonbein, G. Bihlmann, P. vanSon, and H. Sigg, Appl. Phys. Lett. 70, 1602 (1997).

${ }^{10}$ D. H. Zhang, S. F. Yoon, K. Radhakrishnan, and Z. Y. Han, Superlattices Microstruct. 20, 105 (1996).

${ }^{11}$ Y. Fu, N. Li, M. Karlsteen, M. Willander, N. Li, W. L. Xu, W. Lu, and S. C. Shen, J. Appl. Phys. 87, 511 (2000).

${ }^{12}$ F. Szmulowicz, A. Shen, H. C. Liu, G. J. Brown, Z. R. Wasilewski, and M. Buchanan, Phys. Rev. B 61, 13798 (2000).

${ }^{13}$ H. C. Liu, A. G. Steele, M. Buchanan, and Z. R. Wasilewski, J. Appl. Phys. 73, 2029 (1993).

${ }^{14}$ C. C. Chen, H. C. Chen, M. C. Hsu, W. H. Hsieh, C. H. Kuan, S. Y. Wang, and C. P. Lee, J. Appl. Phys. 91, 943 (2003).

${ }^{15}$ C. C. Chen, H. C. Chen, C. H. Kuan, S. D. Lin, and C. P. Lee, Appl. Phys. Lett. 80, 2251 (2002).

${ }^{16}$ C. C. Chen, H. C. Chen, M. C. Hsu, W. H. Hsieh, C. H. Kuan, S. Y. Wang, and C. P. Lee, IEEE J. Quantum Electron. 39, 306 (2003).

${ }^{17}$ J. L. Educato, J. P. Leburton, J. Wang, and D. W. Bailey, Phys. Rev. B 44, 8365 (1991).

${ }^{18}$ S. M. Goodnick and P. Lugli, Phys. Rev. B 37, 2578 (1988).

${ }^{19}$ J. H. Lu, K. J. Wu, K. J. Hsieh, C. H. Kuan, C. W. Yang, S. L. Tu, J. Y. Feng, and T. S. Lay, IEEE J. Quantum Electron. 43, 72 (2007). 